\title{
Steady-state matching and model reduction for systems of differential-algebraic equations
}

\author{
Giordano Scarciotti
}

\begin{abstract}
The problem of model reduction for nonlinear differential-algebraic systems is addressed using the notions of moment and of steady-state response. These notions are formally introduced for this class of systems and families of nonlinear differential-algebraic reduced order models achieving moment matching with additional properties are presented. Stronger results for the special class of linear singular systems are provided. Two simple examples illustrate the proposed technique.
\end{abstract}

\section{INTRODUCTION}

$\mathbf{S}$ INGULAR systems, also known as descriptor systems or differential-algebraic equations ${ }^{1}$ (DAEs) are a class of systems which, combining differential equations with algebraic equations, are used to model constraints and multi-scale behaviors [1]-[3]. In fact, they can be seen as the composition of a fast subsystem (the algebraic part) and a slow subsystem (the differential part). For this reason, systems of differentialalgebraic equations are used to model various behaviors and phenomena, e.g. multi-body systems, electrical networks, chemical processes, social economic systems [1], and to model large interconnected systems, e.g. systems obtained from the application of the Kirchoff's laws or models created by objectoriented modeling languages such as MODELICA [4], [5].

As a result, systems of differential-algebraic equations may have large dimensionality. This has motivated researchers to investigate model reduction techniques. Given a complex high dimensional system, the model reduction problem consists in determining a reduced order model, namely a simple low dimensional mathematical representation with a behavior similar (in a sense to be defined) to the behavior of the complex system. For linear singular systems several approaches have been presented in the last decades. Some of these techniques are based on balanced truncation, see $e . g$. [6]-[12], on interpolation theory, see e.g. [13], and on other variations or ad hoc methods, see e.g. [14]-[19]. More detail and additional references on model reduction of linear singular systems can be found in the survey paper [5]. Conversely, only a few methods to reduce nonlinear singular systems have been proposed. A method based on balanced truncation has been proposed in [4]. Further results have been obtained using piecewise-linear model reduction, see [20]-[22]. Model reduction of nonlinear singular systems in special forms, e.g. bilinear systems, has been investigated in [23], [24]. An approach based on adaptive proper orthogonal

G. Scarciotti is with the Dept. of Electrical and Electronic Engineering, Imperial College London, London, SW7 2AZ, UK, E-mail: g.scarciotti@ic.ac.uk This work was supported in part by Imperial College London under the Junior Research Fellowship Scheme.

${ }^{1}$ Throughout the technical note we use the expressions "singular system" and "system of differential-algebraic equations" interchangeably. decomposition of a related class of distributed parameter systems has been proposed in [25], [26].

In this technical note we present, in a unified framework, a model reduction technique for linear and nonlinear singular systems. Exploiting the connection between moment and steady-state response given in [27], we provide a notion of moment for nonlinear DAEs (Section II-A). We propose families of reduced order models for nonlinear singular systems (Section III-A) and we analyze in detail special families for which additional properties, e.g. strong stability, can be imposed (Section III-B). In addition we specialize the results to linear singular systems, obtaining stronger properties for this particular class (Sections II-B, III-C, III-D).

Preliminary versions of this technical note have been published in [28] and [29]. The additional contributions of the technical note are as follows: we provide all the proofs of the results (in the Appendix); the presentation has been improved, developing the results for nonlinear systems and then deriving the linear framework as a special case; we study the controllability and observability properties of the linear reduced order models; we illustrate the results with two examples, namely we compute the moment in an academic example and we obtain reduced order models for a nonlinear system describing an electrical circuit.

Notation. We use standard notation. $\mathbb{C}_{<0}\left(\mathbb{C}_{0}\right)$ denotes the set of complex numbers with negative (zero) real part. The symbol $\emptyset$ indicates the empty set, $I$ denotes the identity matrix, $\sigma(A)$ denotes the spectrum of the matrix $A \in \mathbb{R}^{n \times n}, \operatorname{rank}(A)$ denotes its rank and $\operatorname{det}(A)$ its determinant. The superscript $\top$ denotes the transposition operator. Given a polynomial $p$, $\operatorname{deg}(p)$ indicates its degree. Given two functions, $f: Y \rightarrow Z$ and $g: X \rightarrow Y$, with $f \circ g: X \rightarrow Z$ we denote the composite function which maps all $x \in X$ to $f(g(x)) \in Z$ as $(f \circ g)(x)=f(g(x))$.

\section{NOTION OF MOMENT FOR CLASSES OF DAES}

In this section we provide the definition of moment for singular systems. We first formulate the notion for nonlinear singular systems. We then conclude the section with an in-depth discussion of the special case of linear singular systems.

\section{A. Moment for nonlinear singular systems}

Consider a nonlinear, single-input, single-output, continuoustime, system described by the equations

$$
E \dot{x}=f(x, u), \quad y=h(x),
$$

with $x(t) \in \mathbb{R}^{n}, u(t) \in \mathbb{R}, y(t) \in \mathbb{R}, E \in \mathbb{R}^{n \times n}$ and $f$ and $h$ smooth mappings. If $E$ is invertible, then equations (1) 
describe a nonlinear system, which we call normal. If $E$ is singular, then system (1) is called singular system. Without loss of generality we assume that $\operatorname{rank}(E)=r<n$ with $E=\operatorname{diag}(I, 0)$. Throughout the technical note we also assume that the initial condition $x(0)$ is consistent, i.e. the initial value problem associated to (1) has at least one solution [2]. Consider a signal generator described by the equations

$$
\dot{\omega}=s(\omega), \quad u=l(\omega),
$$

with $\omega(t) \in \mathbb{R}^{\nu}, s$ and $l$ smooth mappings, and the interconnected system

$$
\dot{\omega}=s(\omega), \quad E \dot{x}=f(x, l(\omega)), \quad y=h(x) .
$$

In addition, suppose that $f(0,0)=0, s(0)=0, l(0)=0$ and $h(0)=0$. The following assumptions and definitions provide a generalization of the notion of moment.

Definition 1: System (2) is observable if for any pair of initial conditions $\omega_{a}(0)$ and $\omega_{b}(0)$, such that $\omega_{a}(0) \neq \omega_{b}(0)$, the corresponding output trajectories $l\left(\omega_{a}(t)\right)$ and $l\left(\omega_{b}(t)\right)$ are such that $l\left(\omega_{a}(t)\right)-l\left(\omega_{b}(t)\right) \not \equiv 0$, i.e. the output trajectories are not indistinguishable, see [30, Definition 3.27].

Assumption 1: The signal generator (2) is observable and neutrally stable. Finally, $\omega(0)$ is almost periodic and such that (2) satisfies the excitation rank condition ${ }^{2}$ at $\omega(0)$.

Assumption 2: The pair $(E, A)$, with $A=\left.\frac{\partial f(x, 0)}{\partial x}\right|_{x=0}$, is strongly stable, i.e. for all $\bar{\lambda} \in \mathbb{C}$ such that $\operatorname{det}(\bar{\lambda} E-A)=0$, $\bar{\lambda} \in \mathbb{C}_{<0}$ and $\operatorname{deg}(\operatorname{det}(\lambda E-A))=r$.

If the system is normal, Assumption 2 reduces to the hypothesis that the zero equilibrium of $\dot{x}=f(x, 0)$ is asymptotically stable in the first approximation. This is a necessary condition for the existence of a center manifold which we intend to exploit to characterize the notion of moment [31]. However, Assumption 2 is stronger than simple stability because it guarantees that all the trajectories of the system exist and are bounded for all $t \geq 0$ (for sufficiently small initial states), i.e. the response of the system is impulse free [34].

Lemma 1: Consider system (1) and the signal generator (2). Suppose Assumptions 1 and 2 hold. Then there is a sufficiently smooth mapping $\pi(\omega)$, with $\pi(0)=0$, locally defined in a neighborhood of $\omega=0$ which solves the partial differentialalgebraic equation

$$
E \frac{\partial \pi}{\partial \omega} s(\omega)=f(\pi(\omega), l(\omega)) .
$$

In addition, for any sufficiently small $x(0)$ and $\omega(0)$, the solution $x(t), \omega(t)$ of (3) exists, is bounded for all $t \geq 0$ and satisfies $\lim _{t \rightarrow \infty} x(t)-\pi(\omega(t))=0$.

Remark 1: Observability and neutral stability of the generator (2) are the only conditions (from Assumption 1) required to prove Lemma 1. However, we want to exclude some "pathological" situations in which the components of the steadystate may be identically zero for selected initial conditions $\omega(0)$ (e.g. we want to exclude the initial condition $\omega(0) \neq 0$ ). For this reason we require the excitability rank condition to be satisfied at an almost periodic $\omega(0)$. This condition

\footnotetext{
${ }^{2}$ See [31, Chapter 8] for the definition of neutral stability. See [32], [33] for the definition of almost periodicity and excitation rank condition.
}

guarantees persistence of excitation of the signal $u$ [32]. For an in-depth discussion of the relation between this condition and the problem of model reduction see [33].

The result of Lemma 1 implies that the interconnected system (3) possesses an invariant center manifold described by the equation $x=\pi(\omega)$ and that this equation expresses the steady-state response of system (1) driven by (2). In analogy with the definition of moment for nonlinear normal systems given in [27] we define the moment for nonlinear singular systems.

Definition 2: Consider system (1) and the signal generator (2). Suppose Assumption 1 holds. The function $h \circ \pi$, with $\pi$ solution of equation (4), is the moment of system (1) at $(s, l)$.

Remark 2: The results can be extended to the class of uncertain systems $E \dot{x}=f(x, u, \widetilde{\eta}), y=h(x, \widetilde{\eta}), u=l(\omega, \widetilde{\eta})$, where $\tilde{\eta} \in \mathbb{R}^{\bar{n}}$ is a vector of unknown parameters of the plant. The results still hold [34, Lemma 8.21] and we can define the parametric moment of the system at $(s, l)$ as the mapping $h \circ \pi$, with $\pi$ solution of the equation $E \frac{\partial \pi}{\partial \omega} s(\omega)=$ $f(\pi(\omega, \widetilde{\eta}), l(\omega, \widetilde{\eta}), \widetilde{\eta})$. This is illustrated in the next example.

Example 1: Consider the nonlinear uncertain singular system described by the equations

$$
\begin{aligned}
\dot{x}_{1}=2 x_{2}+(1+\widetilde{\eta}) x_{3}, & 0=x_{2}+x_{3}, \\
\dot{x}_{2} & =-4 x_{1}-2 x_{2}+x_{4}, \quad 0=-x_{1}-\sin \left(x_{2}\right)+x_{4}+u, \\
y & =x_{4},
\end{aligned}
$$

parametrized in $\widetilde{\eta} \in \mathbb{R}$, and the input

$$
\begin{aligned}
\dot{\omega}_{1} & =\sqrt{2} \omega_{2}, \quad \dot{\omega}_{2}=-\sqrt{2} \omega_{1}, \\
u & =-\left(3-\frac{2}{1-\widetilde{\eta}}\right) \omega_{1}-\frac{2 \sqrt{2}}{1-\widetilde{\eta}} \omega_{2}+\sin \left(\frac{\sqrt{2}}{1-\widetilde{\eta}} \omega_{2}\right) .
\end{aligned}
$$

The pair $(E, A)$ has two finite ${ }^{3}$ eigenvalues $\lambda_{1,2}=-0.5 \pm$ $\sqrt{3 \widetilde{\eta}-2.75}$, both in the left half complex plane if $\widetilde{\eta}<$ 1. Simple computation shows that the mapping $\pi=$ $\left[\begin{array}{llll}\pi_{1} & \pi_{2} & \pi_{3} & \pi_{4}\end{array}\right]^{\top}$ is given by $\pi_{1}=\omega_{1}, \pi_{2}=\frac{\sqrt{2}}{1-\widetilde{\eta}} \omega_{2}$, $\pi_{3}=-\frac{\sqrt{2}}{1-\widetilde{\eta}} \omega_{2}$ and $\pi_{4}=\left(4-\frac{2}{1-\widetilde{\eta}}\right) \omega_{1}+\frac{2 \sqrt{2}}{1-\widetilde{\eta}} \omega_{2}$. For $\widetilde{\eta}<1$ the pair $(E, A)$ is strongly stable, Assumptions 1 and 2 hold, and the steady-state response of system (6) is $\pi(\omega)$. If $\widetilde{\eta}>1$, the mapping $\pi$ is still a solution of the partial differential equation, $\pi_{4}$ is the parametric moment, but we cannot establish a relation with the steady-state response of the system.

Remark 3: Remark 2 and Example 1 show a possible approach to deal with constant uncertain parameters of the system to be reduced. If the uncertain parameter obeys a stochastic distribution, then the techniques presented in [35] to deal with stochastic systems could be extended to the present singular framework. Uncertainties can be addressed also formulating the uncertain system as an inclusion, see [36]. This offers another approach that can be extended to singular systems. Finally, if the model is completely unknown, we can extend to differential-algebraic equations the data-driven techniques based on output measurements which have been presented in [37]-[39].

\footnotetext{
${ }^{3}$ The finite eigenvalues of the pair $(E, A)$ are the zeros of $\operatorname{det}(\lambda E-A)$ for finite values of $\lambda[1]$
} 


\section{B. Moment for linear singular systems}

It is of particular interest to specialize the results we have obtained to the case of linear singular systems. In fact, on one hand, exploiting linearity we can obtain stronger results and provide new insights. On the other hand, since the problem of model reduction for linear singular systems is a classic problem in linear algebra, it is important to relate this framework with the literature.

1) Preliminaries on linear singular systems: in this section we recall some basic results on linear singular systems. Assume system (1) takes the form

$$
E \dot{x}=A x+B u, \quad y=C x,
$$

with $A \in \mathbb{R}^{n \times n}, B \in \mathbb{R}^{n \times 1}$ and $C \in \mathbb{R}^{1 \times n}$. Assume that $u(t)$ is piecewise continuously differentiable.

Definition 3: [1] Let $\mathcal{E} \subset \mathbb{C}$ be the set of complex numbers $\lambda \in \mathbb{C}$ such that $\operatorname{det}(\lambda E-A) \neq 0$. For any two matrices $E$ and $A$, the pencil $(E, A)$ is called regular if $\mathcal{E} \neq \emptyset$.

Lemma 2: [1] The pencil $(E, A)$ is regular if and only if there exist two nonsingular matrices $Q$ and $P$ such that $Q E P=$ $\operatorname{diag}(I, N), Q A P=\operatorname{diag}\left(A_{1}, I\right)$, where $N \in \mathbb{R}^{n_{2} \times n_{2}}$ is nilpotent with degree ${ }^{4} d$ and $A_{1} \in \mathbb{R}^{n_{1} \times n_{1}}$, with $n_{1}+n_{2}=n$.

Assume system (7) is regular, then $Q$ and $P$ can be selected such that system (7) can be written in the so-called first equivalent form [1], namely

$$
\begin{aligned}
& \text { (slow subsystem) } \quad \dot{x}_{1}=A_{1} x_{1}+B_{1} u, \quad y_{1}=C_{1} x_{1}, \\
& \text { (fast subsystem) } \quad N \dot{x}_{2}=x_{2}+B_{2} u, \quad y_{2}=C_{2} x_{2} \text {, } \\
& y=y_{1}+y_{2} \text {, }
\end{aligned}
$$

with the coordinate transformation $\left[\begin{array}{cc}x_{1}^{\top} & x_{2}^{\top}\end{array}\right]^{\top}=P^{-1} x$, where $x_{1} \in \mathbb{R}^{n_{1} \times n_{1}}, x_{2} \in \mathbb{R}^{n_{2} \times n_{2}}, Q B=\left[\begin{array}{ll}B_{1}^{\top} & B_{2}^{\top}\end{array}\right]^{\top}$ and $C P=\left[\begin{array}{ll}C_{1} & C_{2}\end{array}\right]$. The state response of system (7) is given by

$$
\begin{gathered}
x(t)=P\left[\begin{array}{l}
I \\
0
\end{array}\right] \\
\left(e^{A_{1} t} x_{1}(0)+\int_{0}^{t} e^{A_{1}(t-\tau)} B_{1} u(\tau) d \tau\right) \\
-P\left[\begin{array}{l}
0 \\
I
\end{array}\right] \sum_{i=0}^{d-1} N^{i} B_{2} \frac{d^{i}}{d t^{i}} u(t) .
\end{gathered}
$$

For linear singular systems the assumption that the initial condition $x(0)=P\left[\begin{array}{ll}x_{1}^{\top}(0) & x_{2}^{\top}(0)\end{array}\right]^{\top}$ is consistent implies that $x(0)=P\left[\begin{array}{ll}I & 0\end{array}\right]^{\top} x_{1}(0)-\left.P\left[\begin{array}{ll}0 & I\end{array}\right]^{\top} \sum_{i=0}^{d-1} N^{i} B_{2} \frac{d^{i}}{d t^{i}} u(t)\right|_{t=0}$, where $x_{1}(0)$ can be freely selected.

2) Interpolation-based description of moment: in this section we show that the moments of system (7) are uniquely determined by the solution of a generalized Sylvester equation. In the next section we show also that the moments of system (7) are uniquely determined by the steady-state response of the output of a particular interconnected system. Let $W(q)=$ $C(q E-A)^{-1} B=C_{1}\left(q I-A_{1}\right)^{-1} B_{1}+C_{2}(q N-I)^{-1} B_{2}$ be the transfer function associated to system (7) and assume that the system is minimal, i.e., as for normal systems (see [1, Theorem 2-6.3]), controllable and observable.

Definition 4: [40, Chapter 11]) Let $s_{i} \in \mathcal{E}$. The 0-moment of system (7) at $s_{i}$ is the complex number $\eta_{0}\left(s_{i}\right)=C\left(s_{i} E-\right.$

\footnotetext{
${ }^{4}$ A nilpotent matrix is a square matrix $N$ such that $N^{k}=0$ for some positive integer $k$. The smallest such a $k$ is called the degree of $N$.
}

$A)^{-1} B$. The $k$-moment of system (7) at $s_{i}$ is the complex number $\eta_{k}\left(s_{i}\right)=\frac{(-1)^{k}}{k !}\left[\frac{d^{k}}{d q^{k}} W(q)\right]_{q=s_{i}}$, with $k \geq 1$ integer.

The next result, which is a direct extension of [27] (see also [41], [42] for analogous results), gives a relation between the moments and the solution of a generalized Sylvester equation.

Lemma 3: Let $s_{i} \in \mathcal{E}$. Consider system (7), then $\left[\eta_{0}\left(s_{i}\right) \ldots \eta_{k}\left(s_{i}\right)\right]=C \widetilde{\Pi}_{i} \Psi_{k}$, where $\Psi_{k}=$ $\operatorname{diag}\left(1,-1,1, \ldots,(-1)^{k}\right) \in \mathbb{R}^{(k+1) \times(k+1)}$ and $\widetilde{\Pi}_{i} \in \mathbb{R}^{n \times k+1}$ is the unique solution of the generalized Sylvester equation

$$
E \widetilde{\Pi}_{i} \Sigma_{i}=A \widetilde{\Pi}_{i}+B L_{i}
$$

with $L_{i}=\left[\begin{array}{llll}1 & 0 & \ldots & 0\end{array}\right] \in \mathbb{R}^{(k+1)}$ and

$$
\Sigma_{i}=\left[\begin{array}{ccccc}
s_{i} & 1 & 0 & \ldots & 0 \\
0 & s_{i} & 1 & \cdots & 0 \\
\vdots & \vdots & \ddots & \ddots & \vdots \\
0 & \cdots & 0 & s_{i} & 1 \\
0 & \cdots & \cdots & 0 & s_{i}
\end{array}\right] \in \mathbb{R}^{(k+1) \times(k+1)}
$$

Exploiting this lemma, the following result holds.

Theorem 1: Consider system (7) and a set of numbers $s_{i} \in \mathcal{E}$, with $i=1, \ldots, \rho$. Then the moments $\eta_{0}\left(s_{1}\right), \ldots, \eta_{k_{1}-1}\left(s_{1}\right)$, $\ldots, \eta_{0}\left(s_{\rho}\right), \ldots, \eta_{k_{\rho}-1}\left(s_{\rho}\right)$ are uniquely determined by the matrix $C \Pi$, where $\Pi \in \mathbb{R}^{n \times \nu}$ is the unique solution of the generalized Sylvester equation

$$
E \Pi S=A \Pi+B L
$$

with $S \in \mathbb{R}^{\nu \times \nu}$ any non-derogatory ${ }^{5}$ matrix with characteristic polynomial

$$
c(q)=\prod_{i=1}^{\rho}\left(q-s_{i}\right)^{k_{i}},
$$

where $\nu=\sum_{i=1}^{\rho} k_{i}$, and $L \in \mathbb{R}^{1 \times \nu}$ such that the pair $(S, L)$ is observable.

Remark 4: The Sylvester equation (11) is the linear version of the partial differential-algebraic equation (4).

3) Steady-state-based description of moment: we are now ready to present the "steady-state-based" description of moment for linear singular systems. This result links the nonlinear notion given in Section II-A with the linear framework.

Theorem 2: Let $S \in \mathbb{R}^{\nu \times \nu}$ be any non-derogatory matrix with characteristic polynomial (12), with $k_{i}=1$ for all $i=$ $1, \ldots, \rho$. Consider system (7) and suppose that $\sigma(S) \subset \mathbb{C}_{0}$ and $\sigma(A) \subset \mathbb{C}_{<0}$. Consider the interconnection of system (7) with the system

$$
\dot{\omega}=S \omega, \quad u=L \omega
$$

with $L$ and $\omega(0)$ such that the triple $(S, \omega(0), L)$ is minimal. Then the moments $\eta_{0}\left(s_{1}\right), \eta_{0}\left(s_{2}\right), \ldots, \eta_{0}\left(s_{\rho}\right)$ are uniquely determined by the steady-state response of the output of such interconnected system, i.e. $C \Pi \omega$.

Remark 5: In the linear case the moments can be defined as in Definition 4 or equivalently as the matrix $C \Pi$, as established in Theorem 1, or equivalently as steady-state output mapping $C \Pi$, as established in Theorem 2. The definition for nonlinear systems is a nonlinear enhancement of this last characterization

${ }^{5}$ A matrix is non-derogatory if its characteristic and minimal polynomials coincide. 
of moment for linear systems. While in the linear case the three definitions are equivalent, in the nonlinear case the first one does not make sense. For this reason while for linear systems we can define $k$-moments at $s_{i}$, for nonlinear systems we do not define higher order moments.

In the linear framework we can achieve more than the mere restriction of the results of Section II-A. In fact, we now show that the matrix $\Pi$ is partitioned in "slow" and "fast" parts.

Theorem 3: Let $S \in \mathbb{R}^{\nu \times \nu}$ be any non-derogatory matrix with characteristic polynomial (12). Consider system (8) and suppose that $\sigma(S) \cap \sigma\left(A_{1}\right)=\emptyset$. Then the moments $\eta_{0}\left(s_{1}\right), \ldots$, $\eta_{k_{1}-1}\left(s_{1}\right), \ldots, \eta_{0}\left(s_{\rho}\right), \ldots, \eta_{k_{\rho}-1}\left(s_{\rho}\right)$ are uniquely determined

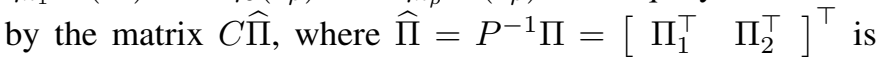
the unique solution of the equations

$$
A_{1} \Pi_{1}-\Pi_{1} S=-B_{1} L, \quad \Pi_{2}=-\sum_{i=0}^{d-1} N^{i} B_{2} L S^{i} .
$$

Remark 6: The contribution of the fast subsystem to $\widehat{\Pi}$ is separated from the contribution of the slow subsystem. Moreover, the condition for uniqueness of $\widehat{\Pi}$ is simplified and it depends only on the eigenvalues of the slow subsystem. This fact is actually expected since in the first equivalent form the $k$-th moment of the system can be written as $\eta_{k}\left(s_{i}\right)=C_{1}\left(s_{i} I-A_{1}\right)^{-(k+1)} B_{1}+C_{2} N^{k}\left(s_{i} N-I\right)^{-(k+1)} B_{2}$ in which $s_{i} N-I$ is full-rank for any $s_{i}$.

This formulation gives high flexibility in maintaining the properties of the fast and slow subsystems. Depending on the particular application the fast subsystem can be preserved, reduced or eliminated independently of the reduction of the slow subsystem. This possibility is analyzed in detail in the next section.

\section{FAMILIES OF REDUCED ORDER MODELS}

In this section we provide families of reduced order models for linear and nonlinear singular systems.

\section{A. Nonlinear singular reduced order models}

We begin giving the definition of reduced order model in the present framework.

Definition 5: Consider system (1) and the signal generator (2). The system described by the equations

$$
\Xi \dot{\xi}=\phi(\xi, u), \quad \psi=\kappa(\xi),
$$

with $\xi(t) \in \mathbb{R}^{\nu}, u(t) \in \mathbb{R}, \psi(t) \in \mathbb{R}, \Xi=\operatorname{diag}(I, 0)$, with $\operatorname{rank}(\Xi)=\bar{r} \leq r$, and $\phi$ and $\kappa$ smooth mappings, is a model of system (1) at $(s, l)$ if system (15) has the same moment at $(s, l)$ as system (1). System (15) is a reduced order model of system (1) at $(s, l)$ if $\nu<n$.

From this definition a rather general result can be presented.

Lemma 4: Consider system (1) and the signal generator (2). Suppose Assumptions 1 and 2 hold. Then system (15) is a model of system $(1)$ at $(s, l)$ if the equation

$$
\Xi \frac{\partial p}{\partial \omega} s(\omega)=\phi(p(\omega), l(\omega))
$$

has a unique solution $p$ such that

$$
h(\pi(\omega))=\kappa(p(\omega))
$$

where $\pi$ is the unique solution of (4).

This claim follows directly from Definition 5 and the definition of moment.

The family of models identified in Lemma 4 has numerous free design "parameters", i.e. $\Xi, \phi$ and $\kappa$, that can be exploited to obtain a convenient representation, as shown in the next section.

\section{B. Nonlinear "identity" family of models}

In the case of differential-algebraic systems it is of interest to have the differential part and the algebraic part separated. At the same time we would like to have an easily tunable parameter which allows to span the family of models. To streamline the presentation of the results we indicate with $\xi_{1}, \bar{s}_{1}(\omega)$ and $\left(\frac{\partial p}{\partial \omega}\right)_{1}$ the first $\bar{r}$ rows of $\xi, s(\omega)$ and $\frac{\partial p}{\partial \omega}$, respectively. For a mapping $\delta: \mathbb{R}^{\nu} \rightarrow \mathbb{R}^{\nu}, \delta_{1}$ indicates the first $\bar{r}$ rows, whereas $\delta_{2}$ denotes the last $\nu-\bar{r}$ rows.

Assumption 3: There exist mappings $\kappa$ and $p$ such that $\kappa(0)=0, p(0)=0, p$ is locally continuously differentiable, equation (17) holds and $p$ has a local inverse $p^{-1}$.

Lemma 5: Consider system (1) and the signal generator (2). Suppose Assumptions 1, 2 and 3 hold. Then the system

$\dot{\xi}_{1}=\bar{s}_{1}(\xi)-\delta_{1}(\xi) l(\xi)+\delta_{1}(\xi) u, \quad 0=-\delta_{2}(\xi) l(\xi)+\delta_{2}(\xi) u$, $\psi=h(\pi(\xi))$,

is a model of system (1) at $(s, l)$ if $\delta$ is an arbitrary mapping such that the partial differential-algebraic equations

$$
\begin{aligned}
\left(\frac{\partial p}{\partial \omega}\right)_{1} s(\omega) & =\bar{s}_{1}(p(\omega))-\delta_{1}(p(\omega)) l(p(\omega))+\delta_{1}(p(\omega)) l(\omega) \\
0 & =-\delta_{2}(p(\omega)) l(p(\omega))+\delta_{2}(p(\omega)) l(\omega)
\end{aligned}
$$

have the unique solution $p(\omega)=\omega$.

The nonlinear differential-algebraic model (18) is parametrized in the mappings $\delta_{1}, \delta_{2}$ which can be used to achieve additional properties. Note that the mapping $p$ may not describe the steady-state response of system (18) unless additional assumptions hold. In fact, from Section II, it follows that we need the additional property that the system be strongly stable.

Lemma 6: Consider system (18) and the signal generator (2). Suppose Assumptions 1 and 2 hold. If $\delta_{1}$ and $\delta_{2}$ are chosen such that the pair $(\Xi, F)$, with

$$
F=\left[\begin{array}{c}
\frac{\partial}{\partial \xi}\left(\bar{s}_{1}(\xi)-\delta_{1}(\xi) l(\xi)\right) \\
-\frac{\partial}{\partial \xi}\left(\delta_{2}(\xi) l(\xi)\right)
\end{array}\right]_{\xi=0},
$$

is strongly stable, then $p(\omega)=\omega$ solves the partial differentialalgebraic equations (19). Moreover, for any sufficiently small $\xi(0)$ and $\omega(0)$, the solution $\xi(t), \omega(t)$ of the interconnection of (18) and (2) exists, is bounded for all $t \geq 0$ and satisfies $\lim _{t \rightarrow \infty} \xi(t)-p(\omega(t))=0$.

This result is a consequence of Lemma 1 applied to the family of reduced order models (18).

Remark 7: The mapping $\delta$ can be selected such that (20) holds. Then the partial differential-algebraic equation (19) is solved and the resulting system (18) is a reduced order model of (1). Moreover, note that condition (20) is a "loose" constraint. In fact, $\delta$ can be selected to satisfy the condition and, at 
the same time, be used to obtain reduced order models with additional properties, see e.g. [27].

Remark 8: To obtain the family of reduced order models (18) we only need to solve equation (4). In general the solution of this partial differential-algebraic equation is difficult to determine analytically. However, the solution can be approximated with numerical methods, see e.g. [34], [37], [38].

Remark 9: Nothing prevents to define a normal reduced order model. Normal reduced order models are actually included in the family of models given so far by simply setting $\Xi=I$. In this case the family (18) becomes

$$
\dot{\xi}=s(\xi)-\delta(\xi) l(\xi)+\delta(\xi) u, \quad \psi=h(\pi(\xi)),
$$

with $\delta$ free, which is the family of reduced order models given in [27].

\section{Linear singular reduced order models}

Since for linear singular systems stronger results can be obtained, we now specialize the results to this class of systems. The possibility of obtaining purely fast or purely slow singular systems is investigated and a "simple" family of linear singular systems is given. In addition, the problem of maintaining controllability and observability properties is discussed.

Definition 6: Consider system (7) and the signal generator (13). The system described by the equations

$$
\begin{aligned}
\dot{\xi}_{1} & =F_{1} \xi_{1}+G_{1} u, \\
\psi_{1} & =H_{1} \xi_{1}, \\
\psi & =\psi_{1}+\psi_{2},
\end{aligned}
$$

where $\xi_{1}(t) \in \mathbb{R}^{\nu_{1}}, \xi_{2}(t) \in \mathbb{R}^{\nu_{2}}$, with $\nu_{1}+\nu_{2}=\nu, \psi_{1}(t) \in \mathbb{R}$, $\psi_{2}(t) \in \mathbb{R}, F_{1} \in \mathbb{R}^{\nu_{1} \times \nu_{1}}, M \in \mathbb{R}^{\nu_{2} \times \nu_{2}}$ nilpotent with degree $\bar{d} \leq d, G_{1} \in \mathbb{R}^{\nu_{1} \times 1}, G_{2} \in \mathbb{R}^{\nu_{2} \times 1}, H_{1} \in \mathbb{R}^{1 \times \nu_{1}}$, and $H_{2} \in$ $\mathbb{R}^{1 \times \nu_{2}}$, is a model of system (7) at $S$, if system (22) has the same moments at $S$ as system (7). System (22) is a reduced order model of system (7) at $S$ if $\nu<n$.

Lemma 7: Consider system (7) and let $S \in \mathbb{R}^{\nu \times \nu}$ be any nonderogatory matrix with characteristic polynomial (12). Assume $\sigma(S) \cap \sigma\left(A_{1}\right)=\emptyset$ and let $L$ be such that the pair $(S, L)$ is observable. Then the system (22) is a model of system (7) at $S$ if there exists a unique solution $\left[\begin{array}{cc}\Gamma_{1}^{\top} & \Gamma_{2}^{\top}\end{array}\right]^{\top}$ of the equations

$$
F_{1} \Gamma_{1}-\Gamma_{1} S=-G_{1} L, \quad \Gamma_{2}=-\sum_{i=0}^{\bar{d}-1} M^{i} G_{2} L S^{i},
$$

such that

$$
C_{1} \Pi_{1}=H_{1} \Gamma_{1}, \quad C_{2} \Pi_{2}=H_{2} \Gamma_{2},
$$

where $\left[\begin{array}{ll}\Pi_{1}^{\top} & \Pi_{2}^{\top}\end{array}\right]^{\top}$ is the unique solution of (14).

This claim follows directly from Definition 6 and the definition of moment.

Similarly to the nonlinear case, there are several parameters that can be exploited to achieve a convenient representation.

\section{Linear "identity" family of models}

In this section we provide a linear equivalent of the family (18). Since for linear singular systems we can use the special structure of $\Pi$ highlighted in (14), it seems natural to select $\Gamma_{1}=I$ keeping $M$ and $G_{2}$ free.

Lemma 8: Consider system (7) and let $S \in \mathbb{R}^{\nu \times \nu}$ be any nonderogatory matrix with characteristic polynomial (12). Assume $\sigma(S) \cap \sigma\left(A_{1}\right)=\emptyset$ and let $L$ be such that the pair $(S, L)$ is observable. Then the system

$$
\begin{aligned}
\dot{\xi}_{1} & =\left(S-G_{1} L\right) \xi_{1}+G_{1} u, \\
M \dot{\xi}_{2} & =\xi_{2}+G_{2} u \\
\psi_{1} & =C_{1} \Pi_{1} \xi_{1} \\
\psi_{2} & =C_{2} \sum_{i=0}^{d-1} N^{i} B_{2} L S^{i}\left(\sum_{i=0}^{\bar{d}-1} M^{i} G_{2} L S^{i}\right) \dot{\xi}_{2}^{-1}, \\
\psi & =\psi_{1}+\psi_{2},
\end{aligned}
$$

where $\left[\begin{array}{ll}\Pi_{1}^{\top} & \Pi_{2}^{\top}\end{array}\right]^{\top}$ is the unique solution of (14), is a model of system (7) at $S$ for any $G_{1}$ such that $\sigma(S) \cap \sigma\left(S-G_{1} L\right)=\emptyset$ and any $G_{2}$ and $M$ such that $\sum_{i=0}^{\bar{d}-1} M^{i} G_{2} L S^{i}$ is invertible.

Remark 10: As noted in [13], in the reduction of non-proper transfer functions $W(s)$ the algebraic part should be maintained unchanged to guarantee that the error between the frequency response of the system to be reduced and the frequency response of the reduced order model stays bounded. This can be achieved in the presented framework setting $\nu_{2}=n-r, \bar{d}=d$. However, in some cases we may not be interested in preserving the rate at which the frequency response grows unbounded, but we may be interested only that it grows unbounded. In other cases, we may be interested only in the output response for low frequencies. The proposed method can deal with these situations leaving to the designer the choice of reducing the fast subsystem, the slow subsystem, or a combination thereof, as shown in the following. Note that a similar discussion can be made for nonlinear singular systems.

Remark 11: Similarly to Remark 9, setting $\nu_{1}=\nu$, we can approximate a singular system with a normal system. Note that this was the first approach attempted [6], although inaccurate since the impulsive characteristic of the singular system is lost [43]. However, in particular settings and as a first approximation it can be useful to approximate a singular system with a normal one. In this case the family (25) becomes

$$
\dot{\xi}=(S-G L) \xi+G u, \quad \psi=C \Pi \xi,
$$

with $G$ free, which is the family of reduced order models given in [27].

Remark 12: Conversely, setting $\nu_{2}=\nu$, we can obtain a completely fast reduced order model. In this case the family (25) becomes

$$
M \dot{\xi}=\xi+G u, \quad \psi=-C \Pi\left(\sum_{i=0}^{\bar{d}-1} M^{i} G L S^{i}\right)^{-1} \xi,
$$

with $M$ nilpotent with degree $\bar{d}$ and $G$ free.

Recall now that system (7) is assumed to be controllable and observable. We conclude this section investigating if these structural properties can be maintained with a specific choice of the free parameters of the reduced order models.

Theorem 4: System (25) has the following properties. 


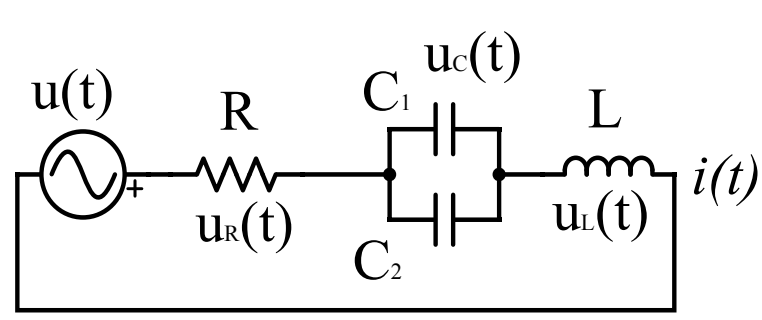

Fig. 1. The electrical circuit in [4].

(C1) The slow subsystem $\dot{\xi}_{1}=\left(S-G_{1} L\right) \xi_{1}+G_{1} u$ is controllable. In this case system (25) is said R-controllable (see [1, Definition 2-2.2]).

(C2) The fast subsystem $M \dot{\xi}_{2}=\xi_{2}+G_{2} u$ is controllable.

(C3) System (25) is controllable.

(C4) System (25) is impulse controllable (see [1, Definition 2-2.3]).

Theorem 5: System (25) has the following properties.

(O1) The slow subsystem $\dot{\xi}_{1}=\left(S-G_{1} L\right) \xi_{1}+G_{1} u, \psi_{1}=$ $C_{1} \Pi_{1} \xi_{1}$ is observable if $G_{1}$ is such that this subsystem has relative degree $\nu_{1}$. In this case system (25) is said R-observable (see [1, Definition 2-3.2]).

(O2) The fast subsystem $M \dot{\xi}_{2}=\xi_{2}+G_{2} u, \psi_{2}=$ $-C_{2} \Pi_{2}\left(\sum_{i=0}^{\bar{d}-1} M^{i} G_{2} L S^{i}\right)^{-1} \xi_{2}$ is observable if $M$ and $G_{2}$ are such that

$$
\operatorname{rank}\left(\left[\begin{array}{c}
M \\
-C_{2} \Pi_{2}\left(\sum_{i=0}^{\bar{d}-1} M^{i} G_{2} L S^{i}\right)^{-1}
\end{array}\right]\right)=\nu_{2} .
$$

(O3) System (25) is observable if the conditions in (O1) and (O2) hold simultaneously.

(O4) System (25) is impulse observable (see [1, Definition 2-3.3]) if $M$ and $G_{2}$ are such that

$\operatorname{rank}\left(\left[\begin{array}{cc}M & I \\ 0 & M \\ 0 & -C_{2} \Pi_{2}\left(\sum_{i=0}^{\bar{d}-1} M^{i} G_{2} L S^{i}\right)^{-1}\end{array}\right]\right)=\nu_{2}+\operatorname{rank}(M)$.

Example 2: We illustrate the results with a simple example. We consider the model studied in [4], which represents the electrical circuit illustrated in Fig. 1. The model is described by the equations

$$
\begin{aligned}
\dot{u}_{C_{1}} & =\frac{1}{2} \frac{i_{1}}{1+10^{-1} u_{C_{1}}}+\frac{1}{2} \frac{i_{2}}{1+10^{-2} u_{C_{1}}}, & & \dot{\Phi}=u_{L}, \\
0 & =u_{R}-5 i_{R}-10 i_{R}^{3}, & & =\Phi-\arctan \left(i_{R}\right), \\
0 & =\frac{i_{1}}{1+10^{-1} u_{C_{1}}}-\frac{i_{2}}{1+10^{-2} u_{C_{1}}}, & & 0=i-i_{1}-i_{2}, \\
0 & =u-u_{R}-u_{C_{1}}-u_{L}, & & y=u_{C_{1}},
\end{aligned}
$$

where $u$ is an ideal voltage source, $u_{R}$ and $i_{R}$ are, respectively, the voltage and the current of the nonlinear resistor, $i_{1}, i_{2}$ and $u_{C_{1}}$ represent, respectively, the currents and the voltage of the capacitors, and $\Phi$ and $u_{L}$ represent, respectively, the saturated flux and the voltage of the inductor. Consider the signal generator (13) with matrices $S=[0,-1 ; 1,0]$ and $L=$ $[0.001,-0.0098]$ which generates an input similar to the one used in [4]. We compute an approximation of the moment $h \circ \pi$ (see [37], [38]), namely $h(\pi(\omega)) \approx 10^{-4}\left(0.0451+9.591 \omega_{1}+\right.$
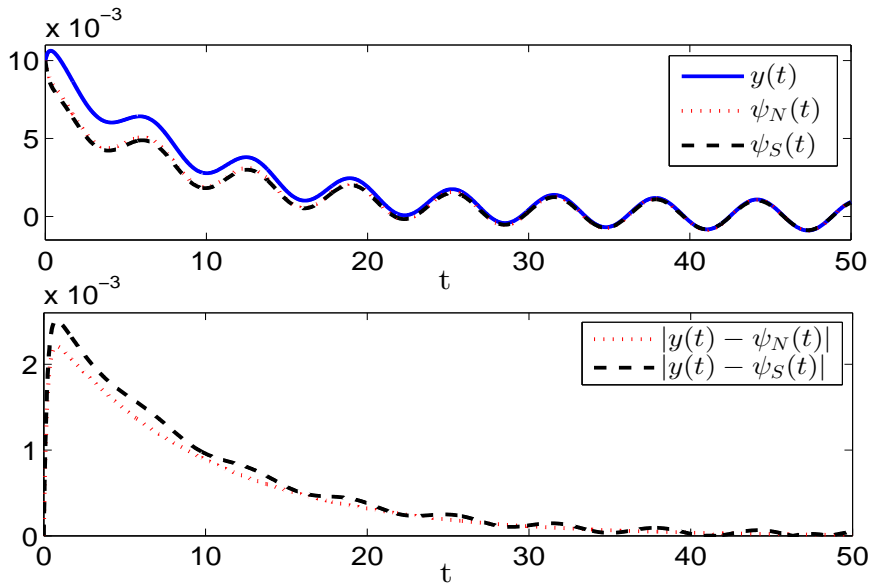

Fig. 2. The top graph shows the time history of the output of system (28) in blue/solid line, of the output of model (29) in red/dotted line and of the output of the model (29) in black/dashed line. The bottom graph shows the absolute errors.

$\left.1.955 \omega_{2}+0.0042 \omega_{1}^{2}-0.0005 \omega_{1} \omega_{2}\right)$. We determine two reduced order models, a normal model described by the equations

$$
\begin{aligned}
\dot{\xi}_{1} & =-0.1021 \xi_{1}+0.0001 \xi_{2}+102.1076 u, \\
\dot{\xi}_{2} & =1.5001 \xi_{1}-4.8979 \xi_{2}-500.0748 u, \\
\psi_{N} & =h(\pi(\xi)),
\end{aligned}
$$

and a singular model described by the equations

$$
\begin{aligned}
\dot{\xi}_{1} & =-0.1028 \xi_{1}+0.0073 \xi_{2}+102.8404 u, \\
0 & =\xi_{1}-9.7944 \xi_{2}-1000 u, \\
\psi_{S} & =h(\pi(\xi)) .
\end{aligned}
$$

Note that both models are linear systems with nonlinear output map (this is expected since the signal generator is linear). The eigenvalues of the dynamic matrix of the model (29) have been set, using the free parameter $G$, as $-0.1021,-4.8979$. These eigenvalues are the eigenvalues of the linearization around $u_{C_{1}}=0, \Phi=0$ of system (28). Similarly, the only finite eigenvalue of model (30) is set as -0.1021 . The resulting two reduced order models are, respectively, asymptotically stable and strongly stable. Fig. 2 (top) shows the time history of the output of system (28) in blue/solid line, of the output of model (29) in red/dotted line and of the output of the model (30) in black/dashed line. The initial conditions of the simulation have been selected as $u_{C_{1}}(0)=0.01, \Phi(0)=0.01$, $i_{R}=0.01, i_{1}=0.005, i_{2}=0.005, u_{L}=-0.06, u_{R}=0.05$, $\omega(0)=\left[\begin{array}{ll}1 & 0\end{array}\right]^{\top}$ and $\xi(0)=\left[\begin{array}{ll}8.6 & 8.8\end{array}\right]^{\top}$. The bottom graph shows the absolute errors $\left|y-\psi_{S}\right|$ (red/dotted line) and $\left|y-\psi_{N}\right|$ (black/dotted line). We see that both the singular model and the normal model are reduced order models of system (28) achieving moment matching in the sense of Definition 5. Hence, from this simulation we are not able to establish which of the two models is preferable.

\section{CONCLUSION}

In this technical note we have extended the notion of moment based on the steady-state response to nonlinear singular systems. We have proposed families of reduced order model for nonlinear and linear singular systems. Connections with the moment 
matching approach based on the interpolation of the transfer function have been drawn. The results have been illustrated by the reduction of a simple electrical circuit.

\section{ACKNOWLEDGMENT}

The author would like to thank the anonymous reviewers for their valuable comments and suggestions to improve the quality of the paper. The author thanks also Dr. A. Astolfi for many helpful comments.

\section{REFERENCES}

[1] L. Dai, Singular Control Systems, ser. Lecture Notes in Control and Information Sciences. Springer Berlin Heidelberg, 1989.

[2] P. Kunkel and V. L. Mehrmann, Differential-algebraic Equations: Analysis and Numerical Solution, ser. EMS textbooks in mathematics. European Mathematical Society, 2006.

[3] R. Riaza, Differential-Algebraic Systems: Analytical Aspects and Circuit Applications. River Edge, NJ, USA: World Scientific Publishing Co., Inc., 2008.

[4] J. Sjöberg, K. Fujimoto, and T. Glad, "Model reduction of nonlinear differential-algebraic equations," in IFAC Symposium Nonlinear Control Systems, Pretoria, South Africa, vol. 7, 2007, pp. 712-717.

[5] P. Benner and T. Stykel, "Model order reduction for differential-algebraic equations: a survey," in (to appear) Surveys in Differential-Algebraic Equations IV, A. Ilchmann and T. Reis, Eds. Berlin, Heidelberg. Springer-Verlag, 2016, preprint MPIMD/15-19, MPI Magdeburg, 2015.

[6] K. Perev and B. Shafai, "Balanced realization and model reduction of singular systems," International Journal of Systems Science, vol. 25 , no. 6, pp. 1039-1052, 1994.

[7] T. Stykel, "Gramian-based model reduction for descriptor systems," Mathematics of Control, Signals and Systems, vol. 16, no. 4, pp. 297-319, 2004.

[8] F. D. Freitas, J. Rommes, and N. Martins, "Gramian-based reduction method applied to large sparse power system descriptor models," IEEE Transactions on Power Systems, vol. 23, no. 3, pp. 1258-1270, Aug 2008.

[9] M. Heinkenschloss, D. C. Sorensen, and K. Sun, "Balanced truncation model reduction for a class of descriptor systems with application to the Oseen equations," SIAM Journal on Scientific Computing, vol. 30, no. 2 , pp. 1038-1063, 2008.

[10] T. Reis and T. Stykel, "Positive real and bounded real balancing for model reduction of descriptor systems," International Journal of Control, vol. 83, no. 1, pp. 74-88, 2010.

[11] T. Stykel, "Balancing-related model reduction of circuit equations using topological structure," in Model Reduction for Circuit Simulation, P. Benner, M. Hinze, and W. E. J. ter Maten, Eds. Dordrecht: Springer Netherlands, 2011, pp. 53-83.

[12] J. Möckel, T. Reis, and T. Stykel, "Linear-quadratic Gaussian balancing for model reduction of differential-algebraic systems," International Journal of Control, vol. 84, no. 10, pp. 1627-1643, 2011.

[13] S. Gugercin, T. Stykel, and S. Wyatt, "Model reduction of descriptor systems by interpolatory projection methods," SIAM Journal on Scientific Computing, vol. 35, no. 5, pp. B1010-B1033, 2013.

[14] F. L. Lewis, M. A. Christodoulou, B. G. Mertzios, and K. Ozcaldiran, "Chained aggregation of singular systems," IEEE Transactions on Automatic Control, vol. 34, no. 9, pp. 1007-1012, Sep 1989.

[15] W. Q. Liu and V. Sreeram, "Model reduction of singular systems," International Journal of Systems Science, vol. 32, no. 10, pp. 12051215, 2001.

[16] J. Wang, Q. Zhang, W. Liu, and V. Sreeram, "Model reduction of singular systems via covariance approximation," in Proceedings of the 2004 American Control Conference, Boston, MA, June, vol. 1, 2004, pp. 90-95

[17] A. B. H. Adamou-Mitiche, L. Mitiche, and V. Sima, "Model reduction for descriptor systems," in First International Symposium on Control, Communications and Signal Processing, March 2004, pp. 827-830.

[18] G. Alì, N. Banagaaya, W. H. A. Schilders, and C. Tischendorf, "Indexaware model order reduction for linear index-2 DAEs with constant coefficients," SIAM Journal on Scientific Computing, vol. 35, no. 3, pp. A1487-A1510, 2013

[19] —, "Index-aware model order reduction for differential-algebraic equations," Mathematical and Computer Modelling of Dynamical Systems, vol. 20, no. 4, pp. 345-373, 2014.
[20] T. Voss, "Model reduction for nonlinear differential-algebraic equations," in Communications to SIMAI Congress, vol. 2, 2007.

[21] T. Voss, A. Verhoeven, T. Bechtold, and J. ter Maten, "Model order reduction for nonlinear differential algebraic equations in circuit simulation," in Progress in Industrial Mathematics at ECMI 2006, L. L. Bonilla, M. Moscoso, G. Platero, and J. M. Vega, Eds. Berlin, Heidelberg: Springer, 2008, pp. 518-523.

[22] B. Bond and L. Daniel, "Stable reduced models for nonlinear descriptor systems through piecewise-linear approximation and projection," IEEE Transactions on Computer-Aided Design of Integrated Circuits and Systems, vol. 28, no. 10, pp. 1467-1480, Oct 2009.

[23] P. Goyal, M. I. Ahmad, and P. Benner, "Krylov subspace-based model reduction for a class of bilinear descriptor systems," Max Planck Institute Magdeburg, Preprint MPIMD/15-07, May 2015.

[24] M. I. Ahmad, P. Benner, P. Goyal, and J. Heiland, "Moment-matching based model reduction for Stokes-type quadratic-bilinear descriptor systems," Max Planck Institute Magdeburg, Preprint MPIMD/15-18, Oct 2015.

[25] D. Babaei Pourkargar and A. Armaou, "Modification to adaptive model reduction for regulation of distributed parameter systems with fast transients," AIChE Journal, vol. 59, no. 12, pp. 4595-4611, 2013.

[26] - "Design of APOD-based switching dynamic observers and output feedback control for a class of nonlinear distributed parameter systems," Chemical Engineering Science, vol. 136, pp. 62-75, 2015.

[27] A. Astolfi, "Model reduction by moment matching for linear and nonlinear systems," IEEE Transactions on Automatic Control, vol. 55, no. 10, pp. 2321-2336, 2010.

[28] G. Scarciotti, "Model reduction for linear singular systems," in Proceedings of the 54th IEEE Conference on Decision and Control, Osaka, Japan, December 15-18, 2015, pp. 7310-7315.

[29] , "Moment matching for nonlinear differential-algebraic equations," in Proceedings of the 55th IEEE Conference on Decision and Control, Las Vegas, NV, USA, December 12-14, 2016, pp. 7447-7452.

[30] H. Nijmeijer and A. van der Schaft, Nonlinear Dynamical Control Systems. Springer, 1990.

[31] A. Isidori, Nonlinear Control Systems, Third ed., ser. Communications and Control Engineering. Springer, 1995.

[32] A. Padoan, G. Scarciotti, and A. Astolfi, "A geometric characterisation of persistently exciting signals generated by autonomous systems," in IFAC Symposium Nonlinear Control Systems, Monterey, CA, USA, August 23-25, 2016, pp. 838-843.

[33] —, "A geometric characterisation of the persistence of excitation condition for the solutions of autonomous systems," To appear on IEEE Transactions on Automatic Control, 2017.

[34] J. Huang, Nonlinear Output Regulation: Theory and Applications, ser. International series in pure and applied mathematics. Philadelphia, PA: SIAM Advances in Design and Control, 2004.

[35] G. Scarciotti and A. R. Teel, "Model order reduction of stochastic linear systems by moment matching," in 20th IFAC World Congress, Toulouse, France, July 9-14 (to appear), 2017.

[36] G. Scarciotti, A. R. Teel, and A. Astolfi, "Model reduction for linear differential inclusions: robustness and time-variance," in Proceedings of the 2017 American Control Conference, Seattle (to appear), 2017.

[37] G. Scarciotti and A. Astolfi, "Data-driven model reduction by moment matching for linear and nonlinear systems," Automatica, vol. 79, pp. 340-351, May 2017.

[38] _ "Model reduction for nonlinear systems and nonlinear time-delay systems from input/output data," in Proceedings of the 54th IEEE Conference on Decision and Control, Osaka, Japan, December 15-18, 2015, pp. 7298-7303.

[39] _ _ "Model reduction for linear systems and linear time-delay systems from input/output data," in 2015 European Control Conference, Linz, July 15-17, 2015, pp. 334-339.

[40] A. Antoulas, Approximation of Large-Scale Dynamical Systems. Philadelphia, PA: SIAM Advances in Design and Control, 2005.

[41] K. Gallivan, A. Vandendorpe, and P. Van Dooren, "Sylvester equations and projection-based model reduction," Journal of Computational and Applied Mathematics, vol. 162, no. 1, pp. 213-229, 2004

[42] K. A. Gallivan, A. Vandendorpe, and P. Van Dooren, "Model reduction and the solution of Sylvester equations," in 17th International Symposium on Mathematical Theory of Networks and Systems, Kyoto, Japan, 2006.

[43] W. Q. Liu and V. Sreeram, "Model reduction of singular systems," in Proceedings of the 39th IEEE Conference on Decision and Control, vol. 3, 2000, pp. 2373-2378.

[44] T. C. Ionescu, A. Astolfi, and P. Colaneri, "Families of moment matching based, low order approximations for linear systems," Systems \& Control Letters, vol. 64, pp. 47-56, 2014 


\section{APPENDIX}

Proof of Lemma 1: Note that this lemma, which characterizes the steady-state response of system (3) in terms of the mapping $\pi$, is a standard result in the literature of output regulation of singular systems. A detailed proof can be found in [34, Lemma 8.9]. As a compromise between completeness and space, we provide a sketch of the proof. Under the stated assumptions the singular system can be rewritten as a normal system. This is proved showing that there exists a change of coordinates such that the algebraic subsystem written in the new coordinates has an obvious solution. The obtained normal system satisfies the assumptions of [31, Proposition 8.1.1]. The proposition establishes that there exists a center manifold which is locally exponentially attractive, is the graph of a mapping solving an equation similar to (4) and can be used to define the steady-state response.

Proof of Lemma 3: Let $\widetilde{\Pi}_{i}=\left[\begin{array}{llll}\widetilde{\Pi}_{i}^{0} & \widetilde{\Pi}_{i}^{1} & \ldots & \widetilde{\Pi}_{i}^{k}\end{array}\right]$ and note that (10) can be rewritten as

$$
\begin{array}{ll}
E \widetilde{\Pi}_{i}^{0} s_{i} & =A \widetilde{\Pi}_{i}^{0}+B, \\
E \widetilde{\Pi}_{i}^{1} s_{i}+E \widetilde{\Pi}_{i}^{0} & =A \widetilde{\Pi}_{i}^{1}, \\
& \vdots \\
E \widetilde{\Pi}_{i}^{k} s_{i}+E \widetilde{\Pi}_{i}^{k-1}=A \widetilde{\Pi}_{i}^{k} .
\end{array}
$$

As a result

$$
\begin{aligned}
\widetilde{\Pi}_{i}^{0} & =\left(s_{i} E-A\right)^{-1} B \\
\widetilde{\Pi}_{i}^{1} & =-\left(s_{i} E-A\right)^{-1} E\left(s_{i} E-A\right)^{-1} B=\left[\frac{d}{d q}(q E-A)^{-1} B\right]_{q=s_{i}} \\
& \vdots \\
\widetilde{\Pi}_{i}^{k} & =\frac{1}{k !}\left[\frac{d^{k}}{d q^{k}}(q E-A)^{-1} B\right]_{q=s_{i}}
\end{aligned}
$$

which proves the claim.

Proof of Theorem 1: Note that (11) has a unique solution if and only if $\prod_{i=1}^{\rho} \operatorname{det}\left(A-s_{i} E\right) \neq 0$. Since the matrix $S$ is non-derogatory, for each distinct eigenvalue $s_{i}$ there exists only one Jordan block $\Sigma_{i}$ of dimension $k_{i}$, i.e. $\bar{\Sigma}=$ $\bar{T} S \bar{T}^{-1}=\operatorname{diag}\left(\Sigma_{1}, \ldots, \Sigma_{\rho}\right)$ is in Jordan form and $L$ becomes $\bar{L}=L \bar{T}^{-1}=\left[\bar{L}_{1}, \ldots, \bar{L}_{\rho}\right]$ where each $\bar{L}_{i}$ block corresponds to a $\Sigma_{i}$ block. We can then consider a pair $\left(\Sigma_{i}, \bar{L}_{i}\right)$ and note that this is observable. It is easy to see that we can find an invertible matrix $\hat{T}$ such that $\Sigma_{i}=\hat{T} \Sigma_{i} \hat{T}^{-1}$ and $L_{i}=\bar{L}_{i} \hat{T}^{-1}$, i.e. it leaves $\Sigma_{i}$ unchanged and transform $\bar{L}_{i}$ in the canonical form in Lemma 3. Let $T=\hat{T} \bar{T}$. Then equation (11) becomes $A \Pi-E \Pi T^{-1} \operatorname{diag}\left(\Sigma_{1}, \ldots, \Sigma_{\rho}\right) T=-B\left[L_{1}, \ldots, L_{\rho}\right] T$. The claim follows multiplying on the right by $T^{-1}$, defining $\Pi=\left[\widetilde{\Pi}_{1}, \ldots, \tilde{\Pi}_{\rho}\right] T$ and applying Lemma 3 to each of the resulting $\rho$ blocks.

Proof of Theorem 2: Consider the interconnection of system (7) with system (13). By the assumptions on the pencil $(E, A)$ and $\sigma(S)$, the interconnected system has a well-defined invariant manifold given by $\mathcal{M}=\left\{(x, \omega) \in \mathbb{R}^{n+\nu}: x=\Pi \omega\right\}$, with $\Pi$ the unique solution of the generalized Sylvester equation (11). We prove now that $\mathcal{M}$ is attractive. Let $z=x-\Pi \omega$. Consider the equation $E \dot{z}=A x+B L \omega-E \Pi S \omega$, which substituting (11), yields $E \dot{z}=A(x-\Pi \omega)=A z$. The solution of this last equation is $z(t)=P\left[\begin{array}{ll}I & 0\end{array}\right]^{\top} e^{A_{1} t} z(0)$. Thus, the output response of the interconnected system is $y(t)=C \Pi \omega(t)+C P\left[\begin{array}{ll}I & 0\end{array}\right]^{\top} e^{A_{1} t}\left(x_{1}(0)-\Pi \omega(0)\right)$. Observing that $C P\left[\begin{array}{ll}I & 0\end{array}\right]^{\top} e^{A_{1} t}\left(x_{1}(0)-\Pi \omega(0)\right)$ describes the transient response (which vanishes exponentially), yields that the steadystate output response of (7) driven by (13) is $C \Pi \omega(t)$. By minimality of $(S, \omega(0), L), C \Pi$ can be uniquely determined by $C \Pi \omega(t)$ computed at $\nu$ sample times $t_{i}$, see [33], [37]. $\square$

Proof of Theorem 3: Let $\bar{x}=\Pi \omega$ and define $\left[\begin{array}{ll}\bar{x}_{1}^{\top} & \bar{x}_{2}^{\top}\end{array}\right]^{\top}=$ $P^{-1} \bar{x}=P^{-1} \Pi \omega=\left[\begin{array}{ll}\Pi_{1}^{\top} & \Pi_{2}^{\top}\end{array}\right]^{\top} \omega$. Substituting these relations in (8) yields

$$
\Pi_{1} S \omega=A_{1} \Pi_{1} \omega+B_{1} L \omega, \quad N \Pi_{2} S \omega=\Pi_{2} \omega+B_{2} L \omega,
$$

from which $\omega$ can be eliminated because the two equations hold for any $\omega$. From the first of these equations we readily obtain the first of equations (14). From the second equation we note that $\Pi_{2}$ is unique if and only if $\operatorname{det}\left(I-s_{i} N\right) \neq 0$, for any $s_{i} \in \sigma(S)$. This holds by definition for any $s_{i} \in \mathbb{C}$ since $N$ is a nilpotent matrix, i.e. $s_{i} N$ has all zero eigenvalues for any $s_{i} \in \mathbb{C}$. Then $\Pi$ is unique if and only if $\sigma(S) \cap \sigma\left(A_{1}\right)=\emptyset$. To obtain the explicit expression of $\Pi_{2}$, we substitute $\bar{x}_{2}=\Pi_{2} \omega$ in the last $n_{2}$ equations in (9) obtaining $\Pi_{2} \omega=-\sum_{i=0}^{d-1} N^{i} B_{2} L \frac{d^{i}}{d t^{i}} \omega$. Observing that $\frac{d^{i}}{d t^{i}} \omega=S^{i} \omega$ yields $\Pi_{2}=-\sum_{i=0}^{d-1} N^{i} B_{2} L S^{i}$, since the equation holds for any $\omega$.

Proof of Lemma 5: Consistently with Lemma 4 and Assumption 3 a family of models that achieves moment matching at $(s, l)$ is described by $\Xi \dot{\xi}=\hat{\phi}(\xi)+\delta(\xi) u$ and $\psi=\kappa(\xi)$, with $\hat{\phi}(\xi)=\left[\Xi \frac{\partial p(\omega)}{\partial \omega}(s(\omega))-\delta(p(\omega)) l(\omega)\right]_{\omega=p^{-1}(\xi)}$, where $p$ is the unique solution of (16) and $\delta$ is a free mapping. Mappings $\kappa$ and $p$ that have the required regularity properties are given by the selection $p(\omega)=\omega$ and $\kappa(\omega)=h(\pi(\omega))$. This yields a family of models described by the equations $\Xi \dot{\xi}=\Xi s(\xi)-\delta(\xi) l(\xi)+\delta(\xi) u$ and $\psi=h(\pi(\xi))$, where $\delta$ is an arbitrary mapping such that equation (16), namely $\Xi \frac{\partial p}{\partial \omega} s(\omega)=\Xi s(p(\omega))-\delta(p(\omega)) l(p(\omega))+\delta(p(\omega)) l(\omega)$, has the unique solution $p(\omega)=\omega$. The claim is proved rewriting these last equations using the definitions given for $\Xi, \xi_{1}, \bar{s}_{1}$, $\delta_{1}, \delta_{2}$ and $\left(\frac{\partial p}{\partial \omega}\right)_{1}$.

Proof of Lemma 8: Consider model (22). $F_{1}$ and $H_{1}$ are selected substituting $\Gamma_{1}=I$ in (23) and (24), respectively. $H_{2}$ is obtained substituting in equation (24) the expressions for $\Gamma_{2}$ and $\Pi_{2}$ given in (23) and (14), respectively.

Proof of Theorem 4: Claim (C1): Since $G_{1}$ is such that $\sigma(S) \cap \sigma\left(S-G_{1} L\right)=\emptyset$, $\dot{\xi}_{1}=\left(S-G_{1} L\right) \xi_{1}+G_{1} u$ is controllable, see [44]. Claim (C2): Recall that $\sum_{i=0}^{\bar{d}-1} M^{i} G_{2} L S^{i}$ is invertible, i.e. $\operatorname{rank}\left(\left[G_{2}\left|M G_{2}\right| \ldots \mid M^{\bar{d}-1} G_{2}\right]\left[L^{\top}\left|S^{\top} L^{\top}\right| \ldots\left|\left(S^{\bar{d}-1}\right)^{\top}\right| L^{\top}\right]^{\top}\right)=$ $\nu_{2}$. Since $(S, L)$ is observable then $\operatorname{rank}\left(\left[G_{2}\left|M G_{2}\right| \ldots \mid M^{\bar{d}-1} G_{2}\right]\right)=\nu_{2}$ which is equivalent to the controllability of the fast subsystem $M \dot{\xi}_{2}=\xi_{2}+G_{2} u[1$, Theorem 2-2.1 (2)(b)]. Claim (C3): (C1) + (C2) imply (C3) [1, Theorem 2-2.1 (3)]. Claim (C4): (C2) implies (C4) [1, p. 37].

Proof of Theorem 5: Claim (O1): This is a direct consequence of [27, Theorem 2]. Claim (O2): This is a direct consequence of [1, Theorem 2-3.1 (3)(d)]. Claim (O3): $(\mathrm{O} 1)+(\mathrm{O} 2)$ imply (O3) [1, Theorem 2-3.1 (4)]. Claim (O4): The condition is obtained after eliminating full rank blocks from the condition given in [1, Theorem 2-3.4 (vii)]. Note that (O2) implies (O4) but $(\mathrm{O} 4)$ does not imply $(\mathrm{O} 2)$. 